\title{
TYPE 2 DIABETICS; FREQUENCY OF VITAMIN D DEFICIENCY
}

1. Assistant Professor Department of Medicine Peoples University of Medical \& Health Sciences for Women Nawabshah, Sindh, Pakistan

2. FCPS (Medicine), (MSc GASRTO) Assistant Professor of Medicine Peoples University of Medical \& Health Sciences for Women Nawabshah, Sindh, Pakistan

3. Post-graduate Student Medical Unit II

Department of Medicine Peoples University of Medical \& Health Sciences for Women Nawabshah, Sindh, Pakistan

Correspondence Address: Dr. Abdul Aziz Sahto FCPS (Medicine), (MSc GASRTO) Assistant Professor of Medicine Peoples University of Medical \& Health Sciences for Women Nawabshah, Sindh, Pakistan draasahito1111@gmail.com

Article received on: 21/07/2016

Accepted for publication:

15/11/2016

Received after proof reading: $18 / 01 / 2017$

\section{INTRODUCTION}

Diabetes mellitus is a chronic metabolic disease characterized by abnormal glucose tolerance. ${ }^{1}$ Type 2 DM results from the failure of cells to response to insulin, sometimes combined with a relative insulin deficiency. Diabetes mellitus is associated with long term complications due to damage of the capillaries causing microangiopathy resulting in Diabetic retinopathy, Diabetic nephropathy, Diabetic neuropathy and some metabolic disturbances. ${ }^{2-4}$

There is recent interest that vitamin $D$ and serum calcium level may have significant relation with diabetes mellitus development as well as glucose homeostasis. Likewise, calcium plays an important role to maintain intracellular insulin-mediated transport of the insulin in insulin dependent tissues such as

\begin{abstract}
Dr. Aamir Shahzad', Dr. Abdul Aziz Sahto², Dr. Asif Ali Memon diagnosed cases of type 2 diabetes mellitus irrespective of age, gender, time since diagnosed, controlled or not controlled and method of treatments like on oral hypoglycemic agents or

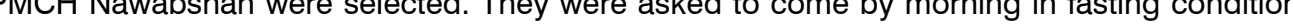
Patients with $25(\mathrm{OH}) \mathrm{D}$ concentration $<20 \mathrm{ng} / \mathrm{ml}$ were labeled as hypovitaminosis D. Data were collected in predefined proforma. SPSS version 20 was used for data entry and for its analysis. Results: Total 126 patients of both gender and age from 30 to 70 years were considered for me patients and 43 female patients. The overall type 2 diabetes mellitus was $68.02 \pm 29.44$ months. It was observed that overall $92.1 \%(N=116)$ found to have deficiency of vitamin D. Conclusion: More than $90 \%$ of the type 2 diabetic patients had deficiency of vitamin $D$ and it is more common among elderly population with male predominance.
\end{abstract}

Key words: $\quad$ Type 2 diabetes mellitus, vitamin D deficiency
Article Citation: Shahzad A, Sahto AA, Memon AA. Type 2 diabetics; frequency of vitamin D deficiency. Professional Med J 2017;24(1):31-35.
DOI: $10.17957 / T P M J / 17.3537$

adipose tissues and skeletal muscle. Those patients who are considered to be high risk for the development of diabetes mellitus found to have low levels of vitamin D. ${ }^{5-7}$

Previously a study conducted that shows if a person takes 800 IU of vitamin D daily and $1200 \mathrm{mg}$ of calcium daily will results in a more than $30 \%$ higher chance of type 2 diabetes mellitus when it was compared with a half dose of vitamin $\mathrm{D}$ and calcium daily.

Besides this there is a lot of other literature which support the association of glucose intolerance with vitamin $D$ deficiency and hypocalcaemia. It has been noticed that blood sugar levels can vary from one season to other season and worsened during the times of winter. Some of the authors correlated this seasonal variation with fluctuations in the vitamin $\mathrm{D}$ concentrations 
due to under exposed to sun light. ${ }^{8-11}$

There is a lack of data about subcontinent showing prevalence of vitamin $D$ deficiency in diabetics. Rational for this study is to document whether local population suffering from diabetes mellitus also has same prevalence of hypovitaminosis D like western part of the world. In case of similarity we can advise calcium and vitamin $D$ supplements with routine therapy for diabetics to achieve better glycemic control as well as calcium homeostasis.

\section{PATIENTS AND METHODS}

This study has been conducted at medicine department of People's Medical University, Shaheed Benazeerabad between the periods of six months. All the diagnosed cases of type 2 diabetes mellitus as determined by fasting blood glucose levels (RBS or urinary glucose are used to monitor therapy rather than for confirm diagnosis, so only those patients who have raised fasting blood glucose level will be included), irrespective of age, gender, time since diagnosed, controlled or not controlled and method of treatments like on oral hypoglycemic agents or insulin therapy were included in study.

Patients suffering from other types of diabetes mellitus such as chronic debilitating diseases i.e. cancer, chronic kidney or liver disease, and bonealtering conditions were excluded from the study.

\section{DATA COLLECTION AND ANALYSIS}

This study commenced after the ethical approval taken from the ethical committee of hospital. Consent was taken from the patients prior to data collection after explain the details. Patients fulfilling the inclusion criteria attending the OPD of medical department of PMCH Nawabshah were selected. They were asked to come by morning in fasting condition and their blood sample was collected for serum vitamin D level. Patients with 25(OH) D concentration $<20 \mathrm{ng} / \mathrm{ml}$ were labeled as hypovitaminosis D. Data were collected in predefined proforma.

The collected data were analyzed using Statistical
Package for Social Science (SPSS) software version 20. Qualitative data were calculated and presented as frequency and percentage while quantitative data were presented as Mean and standard deviation.

\section{RESULTS}

Total 126 patients of both gender and age from 30 to 70 years were included in the study to determine the frequency of Vitamin $D$ deficiency in type-Il diabetic patients. The results showed that there were 83 male patients and 43 female patients, Figure-1.

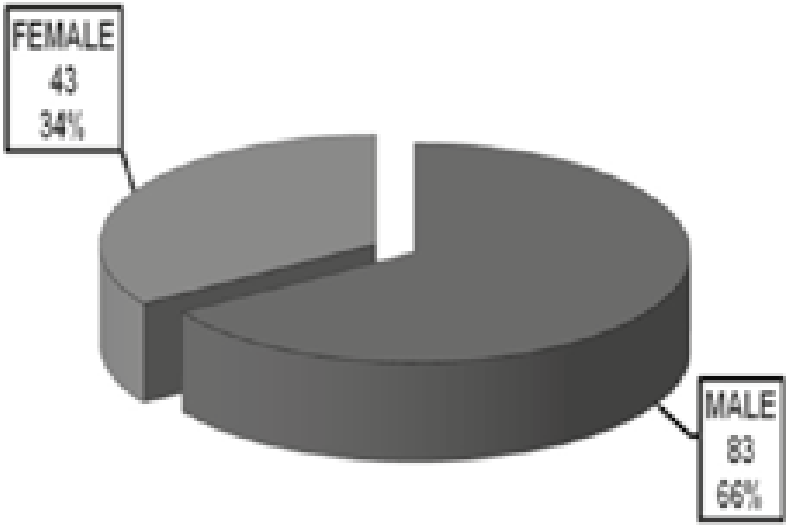

Figure-1. Percentage of patients according to gender

The overall mean age was $48.16 \pm 11.44$ years, with range of 40 (30-70) years. The distribution of age is presented in Figure-2. The age was further stratified in two groups. 68 patients were in $\leq 50$ years of age group and 58 patients were in $\geq 50$ years of age group. The overall mean duration of type 2 diabetes mellitus was $68.02 \pm 29.44$ months, with range of $106(10-116)$ months. Out of total 126 study subject reported with type II diabetes, 43 had controlled diabetes and 83 had uncontrolled diabetes.

The main outcome of the study was the frequency of Vitamin D Deficiency. It was observed that overall $92.1 \%(\mathrm{~N}=116)$ patients were vitamin $\mathrm{D}$ deficient and only $7.9 \%(\mathrm{~N}=10)$ patients were not vitamin $\mathrm{D}$ deficient. The results are also presented in Figure-3.

Stratification with respect to gender, age, duration 
of disease and treatment was done to observe association of these modifiers with the outcome i.e. vitamin $D$ deficiency by applying chi-square test and considered P-value $\leq 0.05$ as significant.

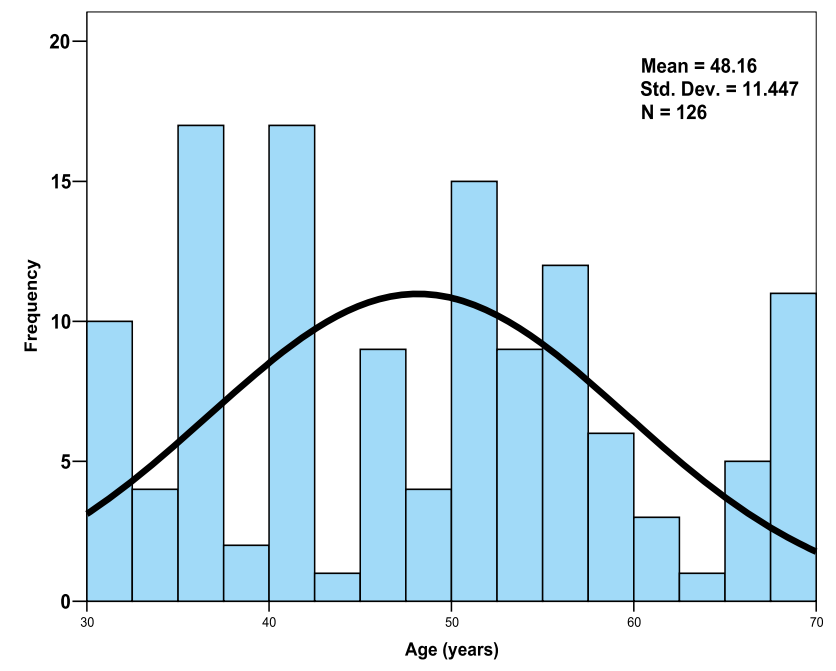

Figure-2. Hitogram presenting distrinbution of age

Figure-3. Percentage of vitamin d deficiency in type 2 diabetic patients

It was found that out of 116 patients, who had positive vitamin $\mathrm{D}$ deficiency, 77 were male patients and 39 were female patients. Among 10 patients with negative vitamin D deficiency, 6 were male patients and 4 were female patients. There was no significant association between the gender and vitamin $D$ deficiency with $p>0.05$, Figure-IV.

\section{DISCUSSION}

Diabetes mellitus is a chronic long standing metabolic condition in which patients suffer

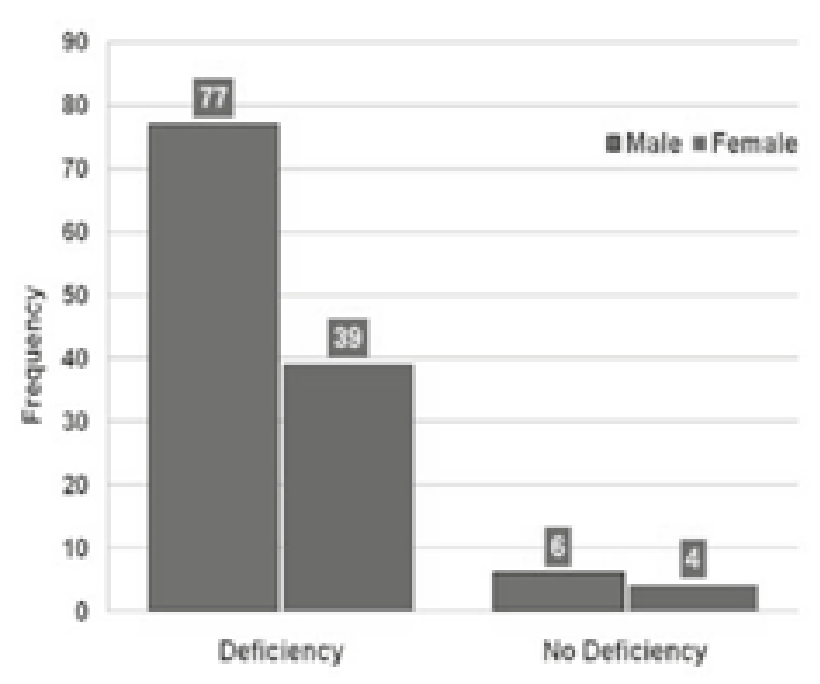

Figure-4. Frequency of vitamin d deficiency in type 2 diabetic patients according to gender

from both mental and physical health problems along with socioeconomic burden. Patients with type 2 diabetes mellitus when unable to control their blood sugar levels will ultimately become depressed and shows disappointment. ${ }^{12,13}$

Pakistani authors has conducted a study to determine the vitamin $\mathrm{D}$ status among them and found that serum $25(\mathrm{OH})$ D of the local Pakistani population was rarely seen abnormal. Through their study we can assume that Pakistani population if are under great exposure to sunlight can maintain a normal levels of serum 25(OH)D concentrations even if the dietary intake is not up to daily requirements as recommended. ${ }^{14}$

Deficiency of vitamin D levels causes depression which is the most important case of development of early type 2 diabetes mellitus now days. A study conducted by Jorden et al. found that supplemental ingestion of vitamin $D$ can lower the risk of depression. ${ }^{15}$

Consistent with these findings which suggest an important relationship in the development of type 2 diabetes mellitus, numerous cross-sectional studies statistically proven that there is a direct relationship with levels of vitamin $D$ and risk of type 2 diabetes mellitus. ${ }^{16}$ In addition, most case-control studies have also found that those individuals who are affected with diabetes mellitus are significantly more likely have a lower levels of 
serum calcifediol in comparison with individuals without type 2 diabetes mellitus. ${ }^{17}$ However, findings have not been entirely consistent, with some studies reporting no association. In addition, a growing number of prospective studies have been conducted with most reporting a significant inverse association of baseline serum 25(OH) D with incident diabetes. This notion was supported in two recent meta-analyses which consisted of around eight to eleven observational studies, according to their findings there is an inverse association between calcifediol with the incident type 2 diabetes mellitus. Though, some studies have also reported no association. ${ }^{16,17}$ These inconsistencies may be due to the use of self-reported vitamin $D$ intake in some studies, a predicted $25(\mathrm{OH}) \mathrm{D}$ score in one study, and the use of self-reported diabetes status to ascertain the primary outcome.

In our study we used $20 \mathrm{ng} / \mathrm{ml}$ value to maintain the cut-off and to label vitamin $D$ deficiency. A total of $92.1 \%$ in our study found to have vitamin D deficiency. These findings when comparing with the findings of previously conducted Indonesian study showed a greater prevalence rate than our study. On the contrary a study conducted by Hollick, has found more than $84 \%$ prevalence of vitamin D deficiency in USA native of Africa. ${ }^{18}$

Gender wise comparison showed that deficiency of vitamin D observed more in males (66.4\%) as compared to females (33.6\%). These variations could be due to their daily work routine and time of exposure to sunlight as females tend to spend their time more at home as compared to males.

\section{CONCLUSION}

The overall prevalence of vitamin $D$ deficiency among type 2 diabetics is more than $90 \%$ and it is observed more common among elderly and males.

\section{Copyright@ 15 Nov, 2016.}

\section{REFERENCE}

1. Olt $\mathrm{S}$. Relationship between vitamin $\mathrm{D}$ and glycemic control in patients with type 2 diabetes mellitus. Int $\mathrm{J}$ Clin Exp Med 2015;8(10):19180-3.
2. Caretta N, de Kreutzenberg SV, Valente U, Guarneri G, Ferlin A, Avogaro A, et al. Hypovitaminosis D is associated with erectile dysfunction in type 2 diabetes. Endocrine 2016 Jan 12.

3. Almurdhi MM, Reeves ND, Bowling FL, Boulton AJ, Jeziorska M, Malik RA. Reduced Lower-Limb Muscle Strength and Volume in Patients With Type 2 Diabetes in Relation to Neuropathy, Intramuscular Fat, and Vitamin D Levels. Diabetes Care 2016 Mar;39(3):441-7.

4. Al-Shahwan MA, Al-Othman AM, Al-Daghri NM, Sabico SB. Effects of 12-month, 2000IU/day vitamin D supplementation on treatment naive and vitamin D deficient Saudi type 2 diabetic patients. Saudi Med J 2015 Dec;36(12):1432-8.

5. Calvo-Romero JM, Ramiro-Lozano JM. Metabolic effects of supplementation with vitamin D in type 2 diabetic patients with vitamin $D$ deficiency. Diabetes Metab Syndr 2015 Oct 9.

6. Heidari B, Nargesi AA, Hafezi-Nejad N, Sheikhbahaei S, Pajouhi A, Nakhjavani M, et al. Assessment of serum 25-hydroxy vitamin D improves coronary heart disease risk stratification in patients with type 2 diabetes. Am Heart J 2015 Sep;170(3):573-9.

7. Calvo-Romero JM, Ramiro-Lozano JM. Vitamin D Levels in Patients With Type 2 Diabetes Mellitus. J Investig Med 2015 Dec;63(8):921-3.

8. Wamberg L, Pedersen SB, Rejnmark L, Richelsen B. Causes of Vitamin D Deficiency and Effect of Vitamin D Supplementation on Metabolic Complications in Obesity: a Review. Curr Obes Rep 2015 Dec;4(4):42940.

9. Flemmer A. [Vitamin D - a valuable protective shield]. Kinderkrankenschwester 2015 May;34(5):192-3.

10. de Court, Mousa A, Naderpoor N, Teede H, de Court, Scragg R. Vitamin D supplementation for the prevention of type 2 diabetes in overweight adults: study protocol for a randomized controlled trial. Trials 2015;16:335.

11. Galesanu C, Mocanu V. Vitamin D deficiency and the clinical consequences. Rev Med Chir Soc Med Nat lasi 2015 Apr;119(2):310-8.

12. Masterson Creber RM, Fleck E, Liu J, Rothenberg G, Ryan B, Bakken S. Identifying the Complexity of Multiple Risk Factors for Obesity Among Urban Latinas. J Immigr Minor Health 2016 May 25.

13. Wennberg AM, Spira AP, Pettigrew C, Soldan A, Zipunnikov V, Rebok GW, et al. Blood glucose levels and cortical thinning in cognitively normal, middle- 
aged adults. J Neurol Sci 2016 Jun 15;365:89-95.

14. Velentzas C, Oreopoulos DG, Brandes L, Wilson DR, Marquez-Julio A. Abnormal vitamin-D levels. Ann Intern Med 1977 Feb;86(2):198.

15. Marriott BM. Vitamin D supplementation: a word of caution. Ann Intern Med 1997 Aug 1;127(3):231-3.

16. Scragg R, Sowers M, Bell C. Serum 25-hydroxyvitamin $D$, diabetes, and ethnicity in the Third National Health and Nutrition Examination Survey. Diabetes
Care 2004 Dec;27(12):2813-8.

17. Scragg R, Holdaway I, Singh V, Metcalf P, Baker J, Dryson E. Serum 25-hydroxyvitamin D3 levels decreased in impaired glucose tolerance and diabetes mellitus. Diabetes Res Clin Pract 1995 Mar;27(3):181-8.

18. Chung JY, Hong SH. Vitamin D status and its association with cardiometabolic risk factors in Korean adults based on a 2008-2010 Korean National Health and Nutrition Examination Survey. Nutr Res Pract 2013 Dec;7(6):495-502.

\section{PREVIOUS RELATED STUDY}

Mohammad Mohsin Rana, Muhammad Saeed Akhtar,,Muhammad Badar bashir, Abaid-ur-Rehman. TYPE 2 DIABETICS COMPONENTS OF THE METABOLIC SYNDROME (Original) Prof Med Jour 13(3) 453-459 Jul, Aug, Sep, 2006.

Mohammad Mohsin Rana, Muhammad Saeed Akhtar, Badar Bashir, Abaid-ur-Rehman. TYPE 2 DIABETICS; THE RELATIONSHIP BETWEEN THE SERUM CHOLESTEROL AND TRIGLYCEROIDS (Original) Prof Med Jour 14(2) 337-343 Apr, May, Jun, 2007.

Azhar Memon, Abdul Raqeeb, Mona Humaira, Haji Khan Khoharo. Type 2 Diabetics; Evaluating serum cobalamin with special reference to dyslipidemia (Original) Professional Med J 2016;23(6): 641-645.

Muhammad Umar Khan, Muhammad Tanveer Alam, Darshan Kumar, Syed Muhammad Adnan, Hala Soomro. Type 2 Diabetic patients; Frequency of self-reported sexual dysfunctions among male (Original) Professional Med J 2016;23(6): 646-654.

Shabnam Naveed, Syed Masroor Ahmed, Ayesha Nageen, Zeeshan Ali, Santosh Kumar, Humaira Zakir, Lubna Ghazi. Type 2 Diabetes; Non alcoholic fatty liver disease (NAFLD) (Original) Professional Med J 2016;23(2): 138-146.

\section{AUTHORSHIP AND CONTRIBUTION DECLARATION}

\begin{tabular}{|c|c|c|c|}
\hline Sr. \# & Author-s Full Name & Contribution to the paper & Author $=$ s Signature \\
\hline 1 & Dr. Aamir Shahzad & $\begin{array}{l}\text { All authors have contributed } \\
\text { equally }\end{array}$ & \\
\hline $\begin{array}{l}2 \\
3\end{array}$ & $\begin{array}{l}\text { Dr. Abdul Aziz Sahto } \\
\text { Dr. Asif Ali Memon }\end{array}$ & & +1 \\
\hline
\end{tabular}

